\title{
The Importance of Including the Geoid in Terrestrial Survey Data Reduction to the Geocentric Datum of Australia
}

\author{
WILL FEATHERSTONE \\ Senior Lecturer in Geodesy \\ School of Surveying and Land Information \\ Curtin University of Technology \\ Perth 6001, Western Australia.
}

\begin{abstract}
The complete reduction of terrestrial survey data to the Geodetic Reference System 1980 (GRS80) spheroid will become an important consideration after the implementation of the Geocentric Datum of Australia (GDA). Three examples are used to illustrate that when survey data reduction does not incorporate the effects of the Earth's gravity field, errors of approximately 11ppm, 200m and 3" can be introduced into terrain distances, astrogeodetically determined coordinates and azimuths respectively.
\end{abstract}

\section{INTRODUCTION}

Historically, geodesists have defined a spheroid to be a close fit to the geoid over each particular region to be surveyed or mapped. This allows several approximations to be made during subsequent survey data reduction. Indeed, this was the case in Australia with the introduction of the Australian National Spheroid or ANS (Bomford, 1967), for which the geoid-ANS separation only reaches $+23 \mathrm{~m}$ (see Figure 1a). This relatively close fit of the ANS to the Australian geoid has considerably simplified the reduction of terrestrial survey data to the Australian Geodetic Datum (AGD). For many practical purposes, the geoid-ANS separation and deflection of the vertical could simply be neglected during survey computations.

However, with the adoption of the Geocentric Datum of Australia (GDA) by the 1st January 2000, the assumptions of zero geoid-spheroid separation and zero deflection of the vertical will not always hold for terrestrial geodetic survey data reduction. It is acknowledged that most conventional geodetic surveys will probably be replaced by the Global Positioning System (GPS). Nevertheless, complete data reduction, which uses a knowledge of the geoid, will still apply to those surveyors who do not have access to GPS survey equipment. Explanations of the definition and implications of the GDA are given elsewhere in the literature; see, for example, the Inter-governmental Committee on Surveying and Mapping (1994), Manning and Harvey (1994), Steed (1995) and Featherstone (1996).

Terrestrial survey measurements are invariably made with respect to the Earth's gravity field because the instruments are aligned with the local gravity vector, by using a spirit 
bubble for example. Therefore, these measurements relate to the geoid, which itself undulates in an irregular fashion. To avoid this complexity of the geoid, a mathematically simple spheroid is chosen to closely fit the geoid, thereby simplifying survey computations. As there is only one geoid, and any number of spheroids can be defined, the survey measurements must be reduced to the particular spheroid on which the datum is based. For instance, survey measurements should be reduced to the ANS when using the AGD.

The GDA will use the spheroid defined by the Geodetic Reference System 1980 or GRS80 (Moritz, 1980). The GRS80 has been defined such that it is a close fit to the geoid on a global scale, and thus does not fit the Australian geoid as well as the ANS. The geoid-GRS80spheroid separation varies from $-32 \mathrm{~m}$ to $+72 \mathrm{~m}$ in Australia (c/f $1 \mathrm{~m}$ to $+23 \mathrm{~m}$ for the geoidANS separation). Figures $1 \mathrm{a}$ and $1 \mathrm{~b}$ illustrate this point, where the difference in the contours of the Australian geoid with respect to the ANS and GRS80 spheroid are due to the different orientation of these spheroids. In Figure 1b, the AUSGEOID93 geoid model (Steed and Holtznagel, 1994) has been augmented by the OSU91A global geoid model (Rapp et al., 1991) in offshore areas.

The previous common assumption of coincidence between the geoid and spheroid is therefore far less valid when using the GDA and GRS80 in Australia. As such, terrestrial geodetic surveys, conducted after the adoption of the GDA, are more likely to require that the geoid-GRS80-spheroid separation and corresponding deflection of the vertical are taken into account. This short paper is intended to illustrate the magnitude of the errors that can occur when terrestrial survey data are reduced to GRS80 instead of the ANS. Examples are given for the effect of the geoid-spheroid separation on measured terrain distances, and the effect of the deflection of the vertical on astrogeodetically determined latitude, longitude and azimuth.

\section{THE GEOID-SPHEROID SEPARATION}

The Australian Height Datum (AHD) will continue to be used for all elevations after the implementation of the GDA. The use of AHD heights alone to reduce survey data to the GRS80 spheroid will usually be inadequate because the geoid-GRS80-spheroid separation is generally greater than the geoid-ANS separation (c/f Figures $1 a$ and $1 b$ ). This is of importance because the omission of six metres in geoid-spheroid separation affects spheroidal distances by approximately one part per million (ppm). Therefore, by ignoring the geoid-GRS80-spheroid separation can contaminate spheroidal distances by between approximately $-5 \mathrm{ppm}$ and $12 \mathrm{ppm}$, whereas by ignoring the geoid-ANS separation only affects spheroidal distances by up to $4 \mathrm{ppm}$.

Some people have argued that the GRS80 spheroid should also be used as the reference surface for elevations. This scenario is impractical. Moreover, it is physically unsound because heights referred to the spheroid alone will allow the possibility that water could appear to flow up-hill, because the physical effect of gravity (via the geoid) is neglected. Also, a proportion of the Australian continent would then have negative heights with 
respect to GRS80, which would certainly cause alarm to the layperson inhabiting these low lying regions. Therefore, the AHD will continue to be used for the foreseeable future.

\section{FIGURE 1}

Contour maps of the Australian geoid with respect to two different spheroids to illustrate their different orientation: a) the Australian National Spheroid (from National Mapping Council, 1986) and b) the Geodetic Reference System 1980 spheroid (after Steed and Holtznagel, 1994, and Rapp et al., 1991).

\section{Terrain Distances}

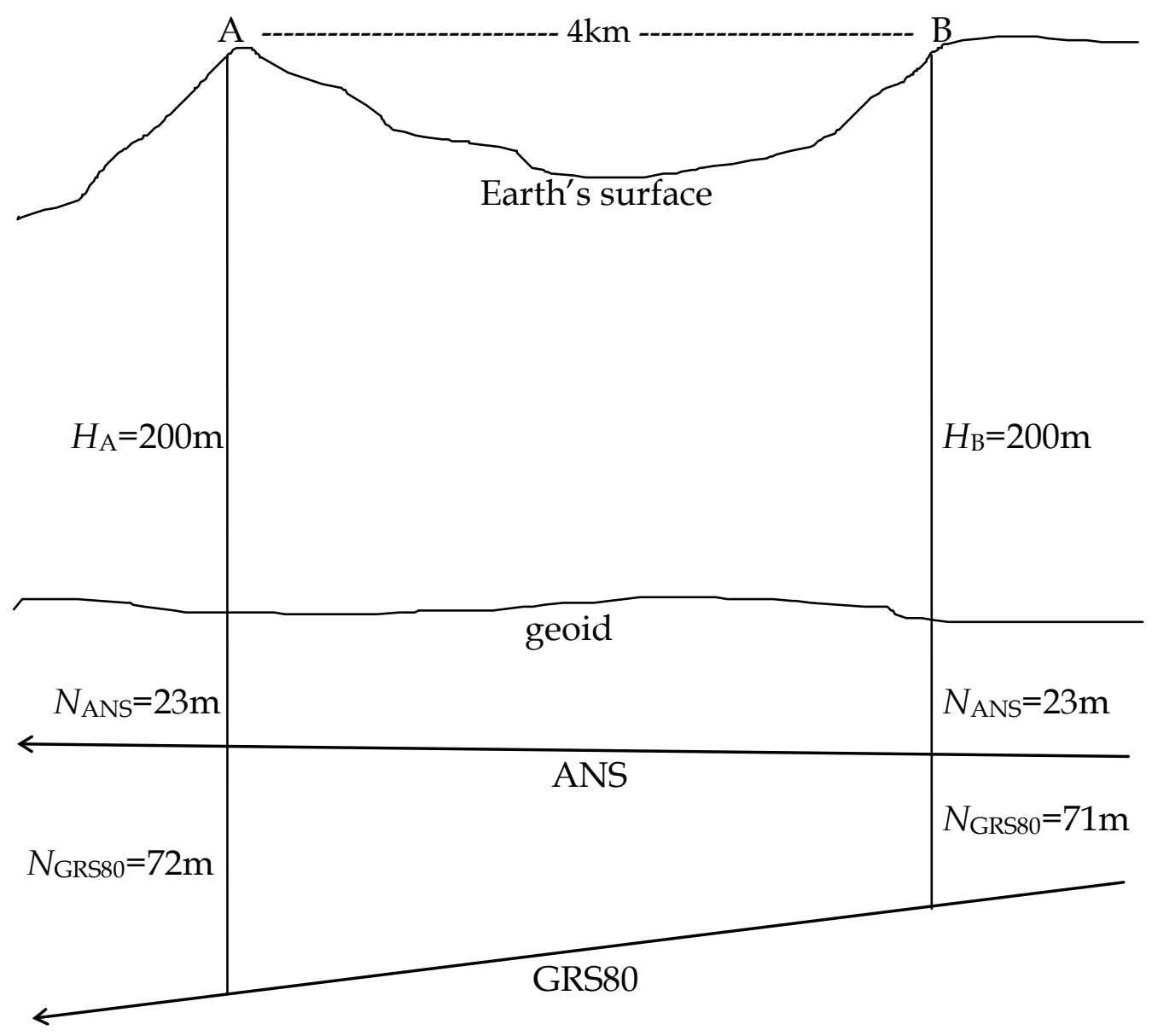

\section{FIGURE 2}

A measured terrain distance of $4 \mathrm{~km}$ and typical station heights in relation to the geoid $(H)$, Australian National Spheroid $\left(h_{\mathrm{ANS}}=H+N_{\mathrm{ANS}}\right)$ and the Geodetic Reference System $1980(h$ $\left.\mathrm{GRS}_{0}=H+N_{\mathrm{GRS}} 0\right)$ spheroid.

To illustrate the effect of neglecting the geoid-spheroid separation on terrestrial survey data reduction, the example of terrain distances is used. In Figure 2, a terrestrial geodetic survey has been used to measure a distance of exactly $4 \mathrm{~km}$ between two stations, $\mathrm{A}$ and $\mathrm{B}$, on the surface of the Earth. This distance has been corrected for instrumental and atmospheric 
refraction effects, and the deflection of the vertical is assumed to be zero at each station. Four cases of survey data reduction are compared:

1. to the ANS using AHD heights only;

2. to the ANS using ANS spheroidal heights;

3. to GRS80 using AHD heights only;

4. to GRS80 using GRS80 spheroidal heights;

where, case 2 is the correct and rigorous approach for data reduction to the ANS and case 4 is the correct and rigorous approach for data reduction to GRS80.

The respective vertical information for stations A and B are: AHD heights $(H)$ of $+200 \mathrm{~m}$ and $+200 \mathrm{~m}$, geoid-ANS separations $\left(N_{\mathrm{ANS}}\right)$ of $+23 \mathrm{~m}$ and $+23 \mathrm{~m}$, and geoid-GRS80-spheroid separations $\left(N_{\mathrm{GRS} 80}\right)$ of $+72 \mathrm{~m}$ and $+71 \mathrm{~m}$. These values have been chosen to produce a realistic, but worst-case, example for survey conditions in Australia. The larger geoidGRS80-spheroid separations over the same baseline are expected because of the poorer fit of and steeper gradient between the geoid and GRS80 spheroid as compared to the ANS (refer to Figure 1). The relations between the AHD height $(H)$ and appropriate spheroidal heights (h) are given by:

$$
\begin{aligned}
& h_{A N S}=H+N_{A N S} \\
& h_{G R S 80}=H+N_{G R S 80}
\end{aligned}
$$

Simple geometry has been used to account for the slope and offset between the terrain and spheroid distances (see section 2.3.4 of National Mapping Council, 1986). The mean radius of curvature of the spheroid is assumed at $30^{\circ} \mathrm{S}$, which corresponds to $R=6,367,450 \mathrm{~m}$ for the ANS (National Mapping Council, 1986) and R=6,367,431m for GRS80 (Murphy, 1994). Table 1 shows the spheroidal distances corresponding to each of the four reductions tested, together with their relative error compared to the exact reductions.

\section{TABLE 1}

Spheroidal distances on the ANS and GRS80 corresponding to a measured terrain distance of $4 \mathrm{~km}$ using approximate (ie. no geoid-spheroid separations) and rigorous (ie. including

\begin{tabular}{|c|c|c|c|c|c|c|c|}
\hline CASE & SPHEROID & $H_{A}$ & & \multicolumn{2}{|c|}{$\begin{array}{c}h_{A} \mathcal{E} h_{B} \\
(\mathrm{~m})\end{array}$} & $\begin{array}{l}\text { SPHEROIDAL } \\
\text { DISTANCE }(\mathrm{m})\end{array}$ & $\begin{array}{c}\text { RELATIVE } \\
\text { ERROR (ppm) }\end{array}$ \\
\hline 1 & ANS & 200 & 200 & \multicolumn{2}{|c|}{--- } & $3,999.8745$ & +3.6 \\
\hline 2 & ANS & \multicolumn{2}{|c|}{--- } & 223 & 223 & $3,999.8600$ & 0 \\
\hline 3 & GRS80 & 200 & 200 & \multicolumn{2}{|c|}{---} & $3,999.8745$ & +11.2 \\
\hline 4 & GRS80 & \multicolumn{2}{|c|}{--- } & 272 & 271 & 3,999.8295 & 0 \\
\hline
\end{tabular}
geoid-spheroid separations) data reduction.

In Table 2, case 2 corresponds to the exact reduction to the ANS and case 4 corresponds to the exact reduction to GRS80 for the same $4 \mathrm{~km}$ baseline, measured on the Earth's surface. 
The difference between these two spheroidal distances is $0.0264 \mathrm{~m}$ (or $\sim 7.6 \mathrm{ppm}$ ), which is solely due to the different size, shape and orientation of the ANS and GRS80 (see Figure 2). The $+3.6 \mathrm{ppm}$ error of case 1 relative to case 2 exceeds the error allowable for most geodetic surveys, which emphasise the importance of using the geoid-ANS separation for current terrain reductions. This geoid-ANS information is available in Annexe E of National Mapping Council (1986).

As may be expected, case 3 introduces a more significant error of $+11.2 \mathrm{ppm}$ into the reduced spheroidal distance relative to case 4 . Therefore, the geoid-GRS80-spheroid separation must be included in the reduction of measured terrain distances for those surveys which require greater accuracy. The geoid-GRS80 separation is currently available in the form of AUSGEOID93 (Steed and Holtznagel, 1994) from AUSLIG in Canberra or the World Wide Web (http://www.auslig.gov.au/geodesy/nvalcomp.htm). Alternatively, the geoid-GRS80 separation can be taken from the OSU91A global geoid model alone (Rapp et al., 1991). In Australia, the geoid-GRS80 separation can usually be ignored in this case along a northwest, south-east band across the continent where it is close to zero (c/f Figure 1b). Conversely, the influence of the geoid on terrain distances becomes most significant in south-western Western Australia and northern Queensland.

\section{THE DEFLECTION OF THE VERTICAL}

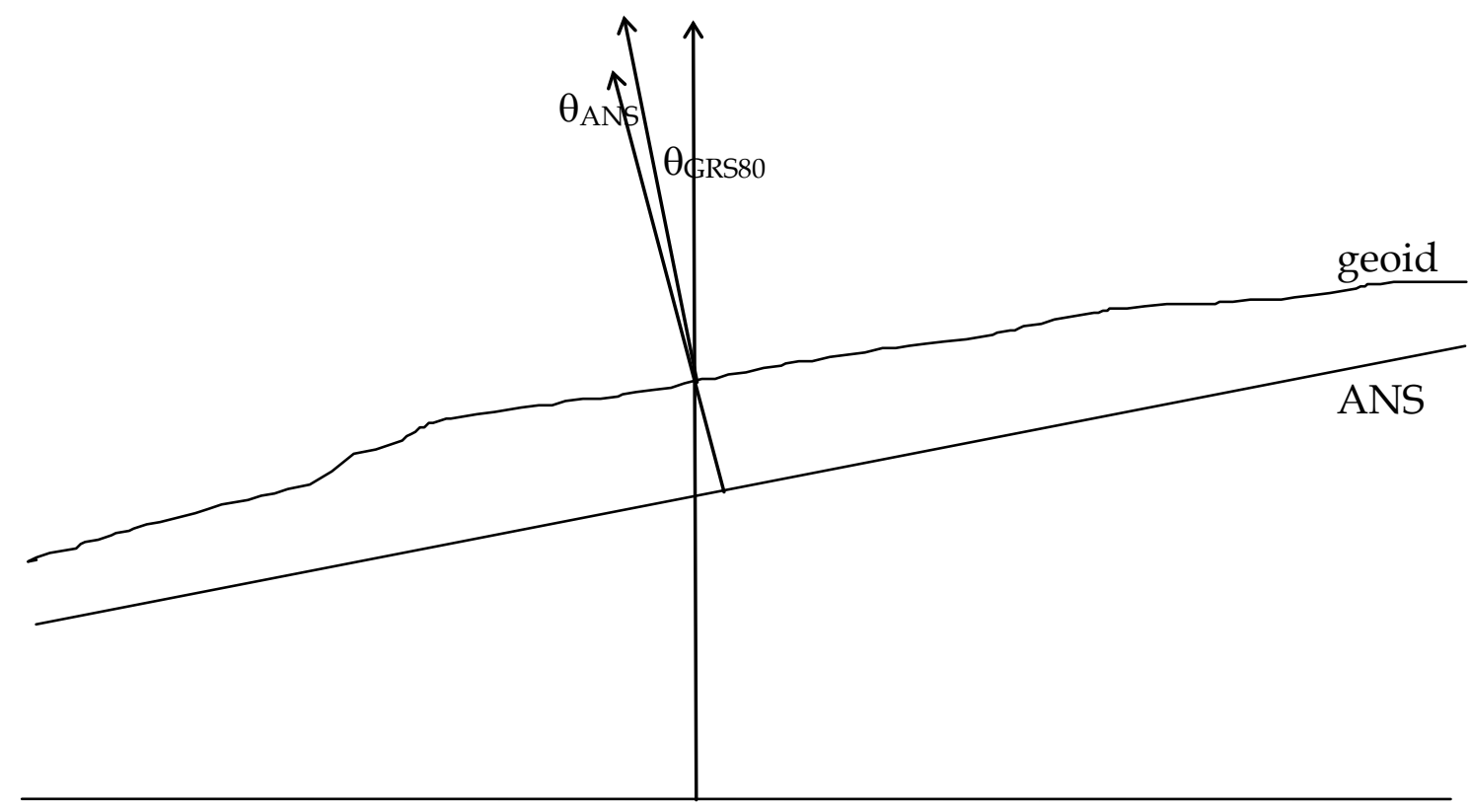

GRS80

\section{FIGURE 3}

A schematic diagram showing the deflection of the vertical referred to the Australian National Spheroid ( $\left.\theta_{\mathrm{ANS}}\right)$ and the Geodetic Reference System 1980 ( $\left.\theta_{\mathrm{GRS}}\right)$ spheroid.

The deflection of the vertical $(\theta)$ is defined as the angular difference between the geoidal normal and the spheroidal normal, and depends directly on the choice of spheroid (see 
Figure 3). Alternatively, it is a measure of the gradient of the geoid with respect to each particular spheroid. Historically, the deflection of the vertical in Australia was minimised by choosing the ANS such that it is parallel to the geoid (Bomford, 1967). As with the geoidspheroid separation, the vertical deflections could be neglected in most instances when surveying using the ANS. However, it becomes more important when reducing terrestrial survey data to the GRS80 spheroid. This is evident in Figure 3, where the vertical deflection is increased for GRS80 because it is not as closely aligned with the Australian geoid as is the ANS.

\section{Astrogeodetic Coordinates and Azimuth}

The total deflection of the vertical $(\theta)$ is most commonly divided into east-west $(\eta)$ and north-south $(\xi)$ components. These can be used to transform astronomical coordinates (latitude $\Phi$ and longitude $\Lambda$ ), as would be determined directly from stellar observations, to geodetic coordinates (latitude $\phi$ and longitude $\lambda$ ) on the local datum. The deflection of the vertical is also used in Laplace's equation for transforming an astronomic azimuth $(A)$, as would be determined directly from stellar or solar observations, to a geodetic azimuth $(\alpha)$. These transformations are given by:

$$
\begin{aligned}
& \phi=\Phi-\xi \\
& \lambda=\Lambda-(\eta / \cos \phi) \\
& \alpha=A-(\eta \tan \phi)
\end{aligned}
$$

In Australia, over a thousand measurements of the deflection of the vertical have been made with respect to the ANS (Fryer, 1971). Their typical magnitude is $\theta=3$ ", whilst the maximum value can reach $\theta=25$ " (see Figure 4). Overall, these deflections point in different directions because of the undulations in the geoid with respect to the ANS (see Figure 1a). Therefore, on average, the deflection of the vertical is expected to be zero because of the overall fit of the ANS to the Australian geoid.

\section{FIGURE 4}

The deflection of the vertical with respect to the ANS at 1133 stations (from Fryer, 1971)

When using the GRS80 spheroid, the deflection of the vertical is systematically increased by approximately $\theta=6$ " in a south-westerly direction, which resolves to $\xi=-4.2^{\prime \prime}$ and $\eta=-4.2^{\prime \prime}$. These values have been estimated by determining the geoid gradient with respect to GRS80 from Figure $1 \mathrm{~b}\left(\sim 104 \mathrm{~m}\right.$ change in $N_{G R S 80}$ over $\left.\sim 3,575 \mathrm{~km}\right)$. The exact size and azimuth of the true deflection of the vertical will vary from place-to-place because of undulations in the geoid. However, these will be common to both the ANS and GRS80. Therefore, it is not necessary to take these into consideration for the purpose of this discussion. 
To illustrate the effect of the deflection of the vertical on the reduction of survey data to the GDA, this systematic increase of approximately $6^{\prime \prime}$ at an azimuth of $225^{\circ}$ is applied to astrogeodetically determined latitude, longitude and azimuth via equations (3), (4) and (5) respectively. The corresponding GDA geodetic data are summarised in Table 2, assuming an astronomical latitude of $\Phi=30^{\circ} 00^{\prime} 00.00^{\prime \prime} S$ and astronomical longitude of $\Lambda=134^{\circ} 00^{\prime}$ $00.00^{\prime \prime} \mathrm{E}$. The deflection of the vertical from the ANS and the curvature of the plumbline are assumed zero at this point. Therefore, the AGD and astronomical coordinates and azimuth are identical.

\section{TABLE 2}

The effect of a systematic increase of 6 " in the deflection of the vertical in a south-westerly direction on astrogeodetic survey data when reduced to the GDA

\begin{tabular}{c|c|ccc}
\hline & ASTROGEODETIC & AGD GEODETIC & GDA GEODETIC & DIFFERENCE \\
\hline latitude & $-30^{\circ} 00^{\prime} 00.00^{\prime \prime}$ & $-30^{\circ} 00^{\prime} 00.00^{\prime \prime}$ & $-29^{\circ} 59^{\prime} 55.76^{\prime \prime}$ & $130.9 \mathrm{~m}$ \\
longitude & $134^{\circ} 00^{\prime} 00.00^{\prime \prime}$ & $134^{\circ} 00^{\prime} 00.00^{\prime \prime}$ & $134^{\circ} 00^{\prime} 04.90^{\prime \prime}$ & $151.2 \mathrm{~m}$ \\
azimuth & $0^{\circ} 00^{\prime} 00.00^{\prime \prime}$ & $0^{\circ} 00^{\prime} 00.00^{\prime \prime}$ & $359^{\circ} 59^{\prime} 57.45^{\prime \prime}$ & $2.55^{\prime \prime}$ \\
\hline
\end{tabular}

An expected observation in Table 2 is that the GDA geodetic coordinates are significantly different to the AGD coordinates. This corresponds to a north-easterly coordinate shift of approximately $200 \mathrm{~m}$. This is similar to the coordinate change that will occur when existing AGD geodetic coordinates are transformed to the GDA. It is therefore interesting to compare the GDA coordinates derived from the AGD coordinates using the seven parameter transformation model to those obtained from astrogeodetic coordinates in conjunction with the deflection of the vertical.

The transformed GDA coordinates are $\phi=29^{\circ} 59^{\prime} 54.84^{\prime \prime} \mathrm{S}$ and $\lambda=134^{\circ} 00^{\prime} 04.83^{\prime \prime} \mathrm{E}$, which differ by approximately $28.5 \mathrm{~m}$ (or $\sim 1^{\prime \prime}$ ) from the astrogeodetically derived GDA coordinates in Table 2. This relatively close agreement vindicates the compatibility between the seven transformation parameters, the fit of the ANS to the Australian geoid, and the Australian gravimetric geoid on GRS80 - AUSGEOID93. A closer agreement could be expected if the true deflections of the vertical were used at this point. Nevertheless, this example is only intended to demonstrate the importance of using the deflection of the vertical to transform astrogeodetic data to the GDA.

The Laplace correction to the astronomic azimuth may introduce a systematic 3" change of orientation of a survey. Again, the need for this correction depends upon the accuracy requirements of the survey, and can usually be neglected for solar determinations of azimuth.

\section{CONCLUSIONS AND RECOMMENDATIONS}

This paper has illustrated the importance of using the geoid-GRS80-ellipsoid separation and corresponding deflection of the vertical when reducing terrestrial survey data to the 
Geocentric Datum of Australia (GDA). Fortunately, the geoid-GRS80-spheroid separation is readily available in the form of AUSGEOID93, which can be purchased for a small fee from AUSLIG, Canberra or accessed via the Internet. However, the deflections of the vertical included with this product have been derived from the OSU91A global geoid model alone, which does not contain the same amount of Australian geoid information as AUSGEOID93. Therefore, to achieve the greatest accuracy, surveyors will have to calculate their own deflections of the vertical from the gridded AUSGEOID93 data using the following formulae:

$$
\begin{aligned}
& \xi=-\frac{\Delta N}{R \Delta \phi} \\
& \eta=-\frac{\Delta N}{(R \cos \phi) \Delta \lambda}
\end{aligned}
$$

where, $R$ is the radius of curvature of the GRS80 spheroid at the point of interest, and $\Delta \mathrm{N}$ refers to the change in the geoid height between the latitudinal and longitudinal geoid grid points. If the point of interest lies between the geoid grid points, some form of interpolation must be used.

It is acknowledged that GPS will most probably be used for the majority of future geodetic surveys. However, geodetic GPS equipment will probably continue to be expensive for some time to come, and not all surveyors will have access to GPS on a routine basis. Therefore, the geoid may become an important tool for surveyors because of the additional reduction of terrestrial survey data to the GDA. Moreover, for those surveyors who rely upon stellar observations to determine azimuth, the deflection of the vertical should be included in the data reduction as its effect is generally greater than the precision of most modern instruments. It is also of similar size to the grid convergence. Therefore, after the implementation of the GDA, the National geoid model, preferably including deflections of the vertical, will become an essential part of the all surveyors' equipment.

The significance of the geoid-GRS80-separations and corresponding deflections of the vertical on terrestrial survey data reduction provides an argument for the continued use of a best fitting (to Australia) spheroid, even in conjunction with the GDA. Whilst avoiding these additional data reductions, the adoption of such a spheroid would be incompatible with GPS. Therefore, the ease of use provided to the surveyor would be far outweighed by the inconvenience caused to the larger population of GPS users.

A final recommendation, which is probably under-way at present, is a revision of the AGD Technical Manual (national Mapping Council, 1986) with the appropriate survey data reduction methods and examples for both terrestrial and GPS surveys on the GDA. Also, the Winter software from AUSLIG should be enhanced to compute deflections of the vertical directly from the gradients of AUSGEOID93 data. 


\section{REFERENCES}

Bomford, A.G. (1967) The geodetic adjustment of Australia, 1963-66. Survey Review, 144, 5271.

Featherstone, W.E. (1996) A revised and updated explanation of the Geocentric Datum of Australia and its effects upon future mapping. The Australian Surveyor, 41(2).

Fryer, J.G. (1971) The geoid in Australia - 1971, Technical report 13, Division of National Mapping, Canberra, ACT.

Inter-governmental Committee on Surveying and Mapping (1994) A new era for Australia. Information Circular, Inter-governmental Committee on Surveying and Mapping, Belconnen, ACT.

Manning, J. and Harvey, W.M. (1994) Status of the Australian geocentric datum. The Australian Surveyor, 39(1), 28-33.

Moritz, H. (1980) Geodetic Reference System 1980. Bulletin Géodésique, 54, 395-405.

Murphy, B (1994) Errata/Addendum - Australian Geodetic Datum Technical Manual. The Australian Surveyor, 39(1), 46-49.

National Mapping Council (1986) The Australian Geodetic Datum Technical Manual. Special Publication no. 10, National Mapping Council of Australia, Canberra, ACT.

Rapp, R.H., Wang, Y.M. and Pavlis, N.K. (1991) The Ohio State 1991 geopotential and sea surface topography harmonic coefficient models, Report 410, Department of Geodetic Science and Surveying, Ohio State University, USA.

Steed, J. and Holtznagel, S. (1994) AHD heights from GPS using AUSGEOID93. The Australian Surveyor, 39(1), 21-27.

Steed, J. (1995) The Geocentric Datum of Australia. Surveying World, 4(1).

\section{BIOGRAPHICAL NOTE}

Dr Will Featherstone is Senior Lecturer in Geodesy at the School of Surveying and Land Information at Curtin University of Technology. Will is a Member of the Institution of Surveyors Australia, the Institution of Engineering and Mining Surveyors and the Mapping Sciences Institute of Australia, and a Fellow of the Royal Astronomical Society. 